\title{
Complex oncologic resection and reconstruction of the scalp: Predictors of morbidity and mortality
}

\author{
Michael G. Tecce, \\ Sammy Othman, \\ Jaclyn T. Mauch, \\ Shelby Nathan, \\ Estifanos Tilahun, \\ Robyn B. Broach, \\ Saïd C. Azoury, \\ Stephen J. Kovach \\ Division of Plastic Surgery, Department \\ of Surgery, University of Pennsylvania, \\ Philadelphia, PA, USA
}

\begin{abstract}
Background: Oncologic resection of the scalp confers several obstacles to the reconstructive surgeon dependent upon patient-specific and wound-specific factors. We aim to describe our experiences with various reconstructive methods, and delineate risk factors for coverage failure and complications in the setting of scalp reconstruction.

Methods: A retrospective chart review was conducted, examining patients who underwent resection of fungating scalp tumors with subsequent soft-tissue reconstruction from 2003 to 2019. Patient demographics, wound and oncologic characteristics, treatment modalities, and outcomes were recorded and analyzed.

Results: A total of 189 patients were appropriate for inclusion, undergoing a range of reconstructive methods from skin grafting to free flaps. Thirty-three patients (17.5\%) underwent preoperative radiation. In all, 48 patients (25.4\%) suffered wound site complications, 25 (13.2\%) underwent reoperation, and $47(24.9 \%)$ suffered from mortality. Preoperative radiation therapy was an independent risk factor for wound complications (odds ratio [OR], 2.85; 95\% confidence interval [Cl], 1.1-7.3; $p=0.028$ ) and reoperations (OR, 4.45; 95\% Cl, 1.5-13.2; $p=0.007)$. Similarly, the presence of an underlying titanium mesh was an independent predictor of wound complications (OR, 2.49; 95\% Cl, 1.1-5.6; $p=0.029$ ) and reoperations (OR, 3.40; 95\% Cl, 1.2-9.7; $p=0.020)$. Both immunosuppressed status $(0 \mathrm{R}, 2.88 ; 95 \% \mathrm{Cl}, 1.2-7.1 ; p=0.021)$ and preoperative radiation therapy (OR, 3.34; 95\% Cl, 1.2-9.7; $p=0.022$ ) were risk factors for mortality.

Conclusion: Both preoperative radiation and the presence of underlying titanium mesh are independent risk factors for wound site complications and increased reoperation rates following oncologic resection and reconstruction of the scalp. Additionally, preoperative radiation, along with an immunosuppressed state, may predict patient mortality following scalp resection and reconstruction.
\end{abstract}

Keywords: Head and neck neoplasms / Microsurgery / Reconstructive surgical procedures / Scalp

\section{INTRODUCTION}

Reconstruction of scalp defects after resection of cutaneous

\footnotetext{
Correspondence: Sammy Othman

Division of Plastic Surgery, University of Pennsylvania, Perelman Center for Advanced Medicine, 3400 Civic Center Blvd, Philadelphia, PA 19104, USA

E-mail: sothman13@gmail.com

This work is accepted for presentation at the 99th Annual American Association of Plastic Surgeons (AAPS) meeting on May 2-5, 2020, in Chicago, IL, USA.

Received May 23, 2020 / Revised June 30, 2020 / Accepted August 18, 2020
}

malignancies can be challenging and requires a considerable degree of surgical planning in order to achieve a satisfactory outcome. Previous studies have developed comprehensive reconstructive algorithms to assist with management of these complex cases [1-3], while other studies have focused on approaches specific to patient risk factors and presentation $[4,5]$. Advances in microsurgery, a more comprehensive understanding of the anatomical blood supply of the scalp, and the advent of new biologic technologies have all provided the reconstruc- 
tive surgeon with more tools to combat these defects [3]. Despite this, the exact role of radiation and other patient-specific factors that confer wound healing complications in the setting of scalp reconstruction have been debated and remain unclear.

When managing cutaneous malignancies of the scalp, surgical excision is often the recommended and preferred initial treatment for cutaneous melanoma, squamous cell carcinoma (SCC), and basal cell carcinoma (BCC), with Mohs micrographic surgery indicated for high-risk cutaneous malignancies in cosmetically sensitive areas [6-8]. Radiation therapy alone may be considered in cases where surgical excision is not possible, contraindicated, or not preferred by the patient after discussion of the risk benefit ratio [6-8]. Although these are effective methods in eradicating malignant lesions and preventing recurrences, they can present complex defects for the reconstructive surgeon. Wide-surgical excision, irradiated and fibrotic native tissue, and prior reconstructive efforts may all limit the options available for definitive coverage [9]. Additionally, radiation to fungating lesions can result in the development of a wound from tumor necrosis that may be very challenging to heal in the radiated field [10]. An additional complicating factor is that patients present for reconstructive consultation with a variety of treatment histories, including before or after radiation therapy, past surgical excision, and prior reconstruction.

Although there exists a general understanding that several factors, such as the presence of radiated/scarred tissue, may cause greater difficulty in achieving reconstructive success, the specific and independent contributions of each have not yet been specifically described. Understanding risk factors and patient characteristics that beget coverage failure may help delineate clear pathways and the appropriate timing of treatment in order to optimize scalp reconstruction. The purpose of this study is to describe our experience with reconstruction of the scalp after resection of cutaneous malignancies, assess outcomes related to different treatment modalities, and identify risk factors for failed reconstruction and their impact on survival.

\section{METHODS}

The authors first obtained institutional review board (IRB) approval. A retrospective chart review was then conducted at a single institution examining patients who underwent resection of a fungating scalp mass with subsequent soft-tissue reconstruction from 2003 to 2019 . The patient population was identified from an unstructured and semi-structured text search engine (PennSeek) using ICD diagnosis codes related to cancer of the scalp and a list of all providers who perform reconstruction at this institution. All subjects were at least 18 years of age.
Subjects were excluded if they had less than a minimum followup of 90 days.

Data on demographic information and patient comorbidities was collected, including age, sex, body mass index (BMI), smoker status, hypertension, diabetes mellitus, peripheral vascular disease, chronic obstructive pulmonary disease, and immune status. Tumor characteristics, wound characteristics, and treatment pathway information were also collected, including defect size, pathohistological tumor type, history of prior reconstruction, preoperative and postoperative radiotherapy and chemotherapy, soft-tissue reconstructive technique, and cranioplasty coverage, where applicable. Finally, data on postoperative course and complications were evaluated, including surgical site infections, wound site complications, flap status, need for reoperation, and final mortality status. Our primary outcomes were rates of wound complication, reoperation, mortality, and free flap failure.

Descriptive statistics were used to categorize frequency and means of all variables. Chi-square testing was used to compare categorical variables, and linear regression was used for comparison of continuous data. A backwards stepwise logistical regression model inclusive of all variables with a $p$-value $\leq 0.1$ on univariate analysis was performed for multivariate analysis. Statistical significance was set at $p \leq 0.05$. Statistical analysis was performed using SPSS version 25 (IBM Corp., Armonk, NY, USA).

\section{RESULTS}

In total, 189 patients met criteria for inclusion. Of this cohort, the average age was $71.5 \pm 15.1$ years, with $71.9 \%$ being male, and a mean BMI of $26.9 \pm 5.8 \mathrm{~kg} / \mathrm{m}^{2}$. Common comorbidities encountered in this group included hypertension (57\%), diabetes mellitus (21\%), immunosuppression (13.2\%), peripheral vascular disease (12\%), and chronic obstructive pulmonary disease (7\%). Nearly half of all patients (48\%) had a positive smoking history (Table 1).

Melanoma was the most frequently encountered histopathological diagnosis (47\%), followed by SCC (24\%), BCC (15\%), and sarcoma (10\%). Overall, 28 patients (14.8\%) received preoperative radiotherapy, $33(18 \%)$ received postoperative radiation, 11 (6\%) received preoperative chemotherapy, and $14(7 \%)$ received postoperative chemotherapy. Reconstructive method varied, with local flaps being the most commonly utilized treatment modality (38.6\%), followed by dermal wound matrices (24.3\%), free flaps (19.5\%), split-thickness skin grafting (14.3\%), and full-thickness skin grafting (3.2\%). Finally, 9.5\% of patients had an underlying titanium mesh implant cranioplasty. All 18 
Table 1. Patient demographics

\begin{tabular}{lc}
\hline Variable & Value $(\mathrm{n}=189)$ \\
\hline Age $(\mathrm{yr})$ & $71.5 \pm 15.1$ \\
Male sex & $139(71.9)$ \\
$\mathrm{BMI}\left(\mathrm{kg} / \mathrm{m}^{2}\right)$ & $26.9 \pm 5.8$ \\
Hypertension & $108(57.1)$ \\
Diabetes mellitus & $39(20.6)$ \\
Smoking status & $91(48.1)$ \\
Peripheral vascular disease & $24(12.7)$ \\
Chronic obstructive pulmonary disease & $13(6.9)$ \\
Immunosuppression & $25(13.2)$ \\
\hline
\end{tabular}

Values are presented as mean \pm SD or number (\%).

BMl, body mass index.

patients with underlying titanium mesh had the mesh placed following oncologic resection (Table 2).

In this cohort, 48 patients (25.4\%) experienced complications. Two patients who underwent skin grafting suffered from complications, for a total complication rate of $6.1 \%(2 / 33)$. These two complications included one superficial infection and one case of minor wound dehiscence, both resolving with conservative treatment. When examining bilayer wound matrices, the complication rate for this modality was $23.4 \%$ (11/46). These 11 complications were four cases of wound dehiscence, two cases of hematoma, one of seroma, and four cases of infection and necrosis. For local flaps, the complication rate was $20.5 \%$ (15/73). These included five instances of wound dehiscence, two seromas, three hematomas, and five cases of infection. The complication rate for free flaps was $54.1 \%(20 / 37)$. These were 10 cases of mesh exposure, two cases of flap failure, three cases of infection, and five cases of wound dehiscence. No individual factors were associated with skin graft complications, but preoperative radiation was associated with flap complications, and titanium mesh was associated with free flap complications $(p<0.05$ for these factors).

In summation, the overall types and frequencies of complications included wound dehiscence (15/48, 31.3\%), necrosis/infection $(13 / 48,27.1 \%)$, mesh exposure $(10 / 48,20.8 \%)$, hematoma $(5 / 48,10.4 \%)$, seroma $(3 / 48,6.3 \%)$, and flap failure $(2 / 48$, $4.2 \%)$. Multivariate analysis revealed that preoperative radiotherapy was significantly predictive of postoperative surgical site complications (odds ratio [OR], 2.85; 95\% confidence interval [CI], 1.1-7.3; $p=0.028$ ). The presence of titanium mesh was also a significant predictor of wound site complications (OR, 2.49; 95\% CI, 1.1-5.6; $p=0.029$ ) (Table 3).

Reoperative rates were also examined. In total, 25 patients underwent reoperation rate for a total of a $13.2 \%$ reoperation rate. Patients who received skin grafting did not undergo any reop-
Table 2. Oncological history and repair characteristics

\begin{tabular}{lc}
\hline Variable & Value \\
\hline Previous reconstruction & $76(40.2)$ \\
Defect size $\left(\mathrm{cm}^{2}\right)$ & $76.7 \pm 87.9$ \\
Histology & \\
Melanoma & $89(47.1)$ \\
Squamous cell carcinoma & $46(24.3)$ \\
Basal cell carcinoma & $28(14.8)$ \\
Sarcoma & $18(9.5)$ \\
Other & $8(4.2)$ \\
Adjunct therapy & \\
Preoperative radiation & $28(14.8)$ \\
Postoperative radiation & $33(17.5)$ \\
Preoperative chemotherapy & $11(5.8)$ \\
Postoperative chemotherapy & $14(7.4)$ \\
Reconstructive strategy & \\
Split-thickness skin graft & $27(14.3)$ \\
Full-thickness skin graft & $6(3.2)$ \\
Dermal wound matrix & $46(24.3)$ \\
Local tissue rearrangement & $73(38.6)$ \\
Free flap & $37(19.5)$ \\
Titanium mesh & $18(9.5)$ \\
\hline
\end{tabular}

Values are presented as number (\%) or mean \pm SD.

Table 3. Multivariate analysis for outcomes

\begin{tabular}{lccc}
\hline Outcome & Frequency (\%) & OR (95\% Cl) & $p$-value \\
\hline Wound complication & $48(25.4)$ & & \\
$\quad$ Preoperative radiotherapy & & $2.85(1.1-7.3)$ & 0.028 \\
$\quad$ Titanium mesh & & $2.49(1.1-5.6)$ & 0.029 \\
$\begin{array}{l}\text { Reoperation } \\
\quad \text { Preoperative radiotherapy }\end{array}$ & & $4.45(1.5-13.2)$ & 0.007 \\
$\quad$ Titanium mesh & & $3.40(1.2-9.7)$ & 0.020 \\
Mortality & $47(24.9)$ & & \\
$\quad$ Preoperative radiotherapy & & $3.34(1.2-9.7)$ & 0.022 \\
Immunosuppressed status & & $2.88(1.2-7.1)$ & 0.021 \\
\hline
\end{tabular}

$\mathrm{OR}$, odds ratio; $\mathrm{Cl}$, confidence interval.

erations ( $0 \%$, while $6.5 \%$ of patients who underwent coverage with a bilayer wound matrix underwent reoperation due to two cases of infection and necrosis and two cases of wound dehiscence $(4 / 46,6.5 \%)$. The total reoperative rate for patients treated with a local flap was $9.6 \%(5 / 73)$. These were due to three cases of dehiscence and two cases of infection. Patients treated with a free flap had a reoperation rate of $43.2 \%$ (16/37). These were for 10 cases of mesh exposure, two cases of flap failure, three cases of wound dehiscence, and one infection. Preoperative radiation was associated with reoperation for flaps, while titanium mesh was associated with reoperation for free flaps $(p<0.05)$. 
In total, these reoperations were due to mesh exposure (10/25, $40 \%)$, dehiscence $(8 / 25,32 \%)$, necrosis/infection $(5 / 25,25 \%)$, and flap failure $(2 / 25,8 \%)$. On multivariate analysis, both preoperative radiotherapy (OR, 4.45; 95\% CI, 1.5-13.2; $p=0.007$ ) and the presence of an underlying titanium mesh (OR, 3.40; $95 \%$ CI, 1.2-9.7; $p=0.020$ ) were found to be significant risk factors for reoperation (Table 3). Age, sex, comorbidities, histopathological diagnosis, recurrence history, postoperative radiation, chemotherapy, and reconstructive modality were all not found to have any significant association with either reoperative rates or wound complications.

Of the 37 patients who underwent reconstruction with free tissue transfer, two patients encountered complete failure $(5.4 \%$ of all patients with a free flap). The first patient suffered from complete failure of his anterolateral thigh flap postoperatively due to pressure necrosis of the arterial anastomosis complicated by the presence of an underlying titanium mesh. This patient was taken back to the operating room for excision of his nonviable flap, with a new attempt for wound coverage performed with a latissimus dorsi flap. The patient recovered without further complications. A second patient also suffered complete loss of his anterolateral thigh flap in the immediate postoperative period due to arterial thrombosis. The flap was found to be non-viable; however, the patient soon expired due to his complex medical history before repair could be undertaken. No variables were found to be significantly associated with flap loss in this group. No flap type, including free and local flaps, was associated with failure.

When examining all-cause mortality, 47 patients (24.9\%) were found to be deceased on last follow-up. Preoperative radiother-

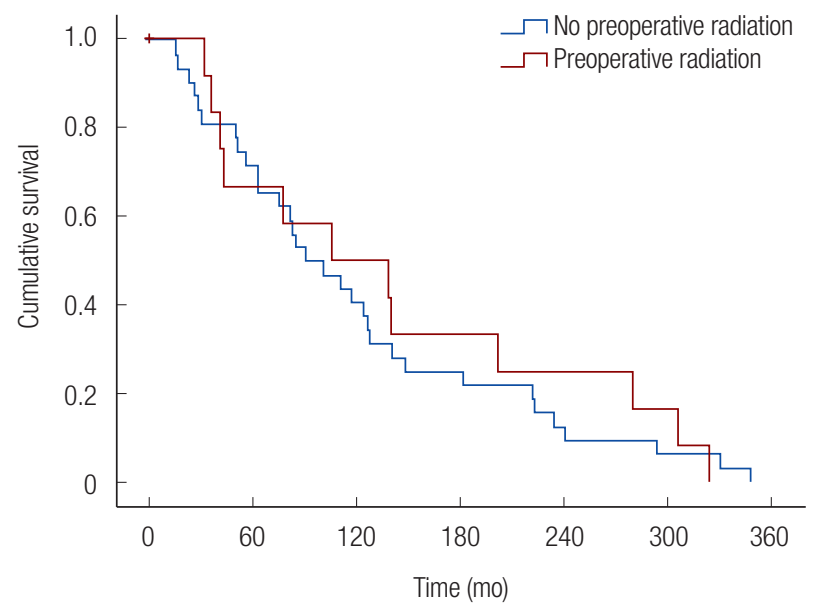

Fig. 1. Radiated and non-radiated patient time to mortality. KaplanMeier survival curve of deceased patients. The mean survival time of patients with preoperative radiation was $171.6 \pm 47.3$ months, while those who did not receive radiation survived a mean of $231.2 \pm 110.8$ months $(p=0.658)$. apy (OR, 3.34; 95\% CI, 1.2-9.7; $p=0.022)$ and immunosuppressed status (OR, 2.88; 95\% CI, 1.2-7.1; $p=0.021$ ) were both predictive of mortality on multivariate analysis (Table 3 ).

Overall survival for patients who underwent preoperative radiation compared to those who did not undergo preoperative radiation was further examined. The mean survival time for patients who received preoperative radiation $(25.5 \%$ of deceased patients; mean survival time: $171.6 \pm 47.3$ months) was similar to the survival time of those who did not receive radiation (74.5\% of deceased patients; mean survival time: $231.2 \pm$ 110.8 months) $(p=0.658)$ (Fig. 1).

\section{DISCUSSION}

Achieving successful wound coverage following resection of cutaneous scalp malignancies can present unique challenges. Although a breadth of literature has examined management pathways, few studies to date have specifically delineated risk factors and patient characteristics that are predictors of morbidity and mortality. The aim of this study was to describe our experiences with reconstruction of the scalp and calvarium, and evaluate risk factors for complications across multiple methods of reconstructing defects that have resulted from the excision of large fungating malignancies of the scalp. In our experience, the use of local flaps (Fig. 2) and dermal wound matrices comprised greater than half of the reconstructive methods utilized.

In the present study, preoperative radiation therapy was found to be a significant risk factor for developing a wound complication, as well as the likelihood of requiring a reoperation. Chao et al. [5] reviewed 138 scalp procedures and found preoperative
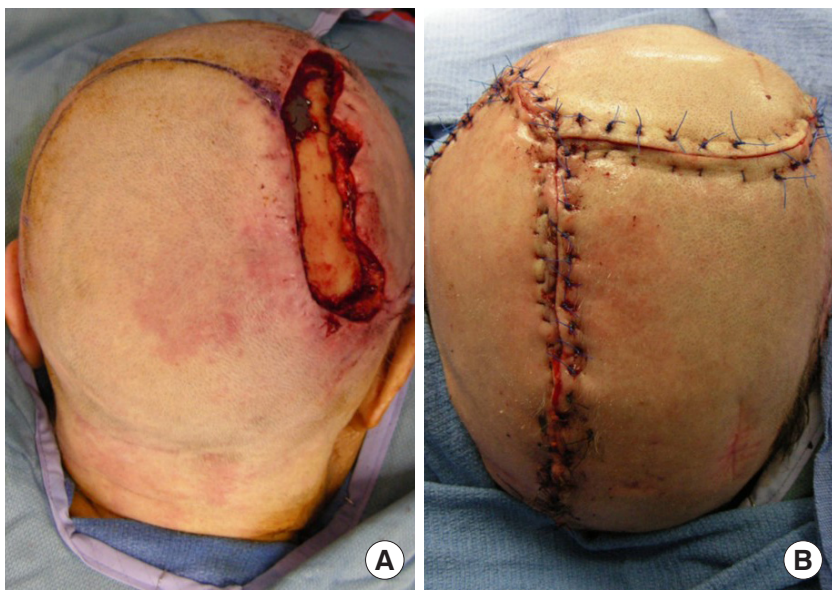

Fig. 2. An elderly male patient presented with biopsy confirmed squamous cell carcinoma of the scalp. (A) Following resection, an intraoperative view exposed calvarium amenable to local flap reconstruction. (B) Complete scalp reconstruction following the harvest of a scalp rotational flap. 
scalp radiation to be associated with the risk for developing perioperative complications. Janus et al. [10] also observed that preoperative radiation, in addition to larger/deeper wounds and immunosuppressed status, conferred greater risk of complications in oncologic reconstruction. Scalp irradiation can produce skin fibrosis and lead to intrinsic changes to the integument, creating a propensity for nonhealing wounds [11]. Such changes compromise the underlying vascular supply, prevent formation of an ingrowing vascular bed, and beget difficulties for wound healing in the setting of damaged adjacent tissue [12]. This is a critical point, as local flaps or wound closures under tension that may otherwise survive in a nonirradiated scalp may be more likely to result in complications in patients with a history of radiation. Further, the viability of free tissue transfer is critically dependent upon the availability of arterial inflow and venous outflow. Vessels may be damaged in the setting of preoperative radiation, and may make the microvascular reconstruction more challenging, potentially creating the need for interposition vein grafts. Given the apparent deleterious effects of preoperative radiation and increased risk of surgical morbidity, the authors sought to examine if preoperative radiation was associated with any improvement in survival compared to no radiation. All-cause mortality survival time, however, was similar between those two groups.

Several studies have examined the risk benefit ratio associated with preoperative radiation of angiosarcoma of the scalp and its impact on survival [13-15]. In a meta-analysis by Shin et al. [16], it was found that radiation therapy did not have a significant effect on survival in head and neck angiosarcoma. In our study, the majority of patients held a diagnosis of melanoma,
SCC, or BCC. This study showed that not only was preoperative radiation predictive of increased complications and mortality, but also had no effect on survival times. Other studies have examined outcomes of scalp melanoma and nonmelanoma cancers based on tumor staging. Terakedis et al. [17] showed that two-thirds of patients receiving preoperative radiation experienced recurrent disease in high-risk melanoma, though some studies have reported important radiation benefits dependent upon the specific cancer histology, stage, and subset patient population [17-20].

Anecdotally, it is the authors' experience that bilayer wound matrices undergo greater complications when lesions are radiated preoperatively. For these reasons, the authors opt for flap coverage, where possible, in patients with previously radiated wounds. Patients with small, uncomplicated lesions (less than $100 \mathrm{~cm}^{2}$ ) usually undergo local flaps, while larger, more complicated lesions undergo free flap coverage. However, in uncomplicated lesions in patients with no radiation history, the authors believe bilayer wound matrices are a strong option (Fig. 3). Thus, in consideration of the risk profile that accompanies preoperative radiation as discussed above, patients should be thoroughly counseled regarding the risk benefit ratio, and consideration should be given to reconstruct large radiated defects with free flaps in order to provide additional healthy soft-tissue bulk [10,21]. Our preferred approach is for formal surgical resection until negative margins are achieved and confirmed by pathological analysis. This is then followed by reconstruction, with the addition of postoperative radiation for patients with high-risk lesions.

Importantly, we also note that immunosuppression was asso-

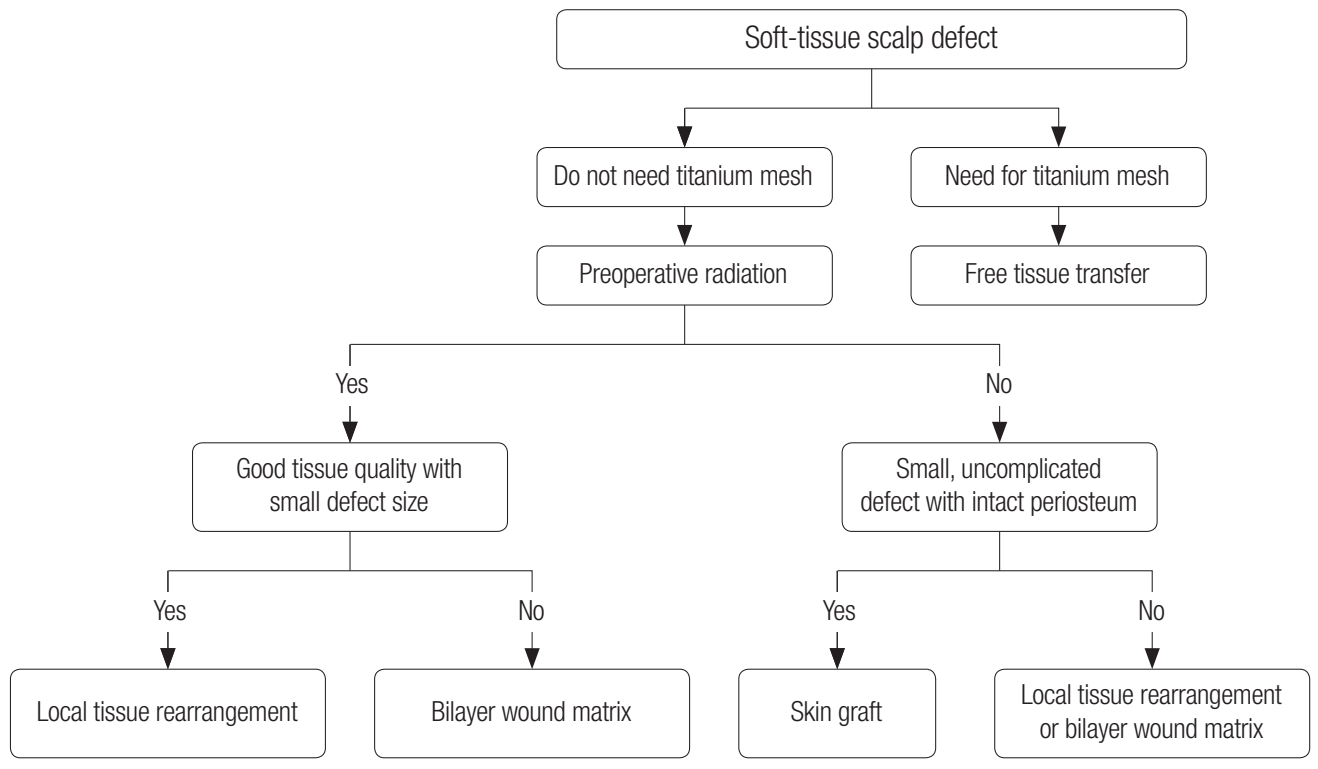

Fig. 3. Algorithm for soft-tissue reconstruction following oncologic resection. 
ciated with mortality. Chronic immunosuppressed status is associated with a host of morbidities, including cardiovascular, endocrine, and oncologic side effects, amongst many others $[22,23]$. Oncologic patients may be immunosuppressed due to their condition, and their risk of wound healing difficulties may consequently be magnified. Other patient comorbidities, such as smoking and increased age, have been previously associated with wound healing difficulties in the context of head and neck reconstruction [24]. Although this was not shown in this study, it is necessary to keep total patient comorbidities in context when considering wound reconstruction.

Titanium mesh provides a well-studied, effective coverage technique for the central nervous system in the setting of calvarial resection $[25,26]$. Although the mesh is efficacious for this purpose, previous studies have determined that underlying mesh in the setting of scalp reconstruction increases the risk for wound complications $[27,28]$ This study also showed that titanium mesh increases not only the risk of complications, but also the propensity for the need for reoperation. We hypothesize that the presence of underlying mesh can compromise the underlying blood supply, which is critical for flap survival and flap efficacy [27-29]. This may consequently predispose ischemic tension, wound complications, and, ultimately, coverage failure, resulting in mesh exposure. Previous research also indicates that titanium mesh may cause atrophy of the overlying soft-tissues. Elderly patients often present with fragile skin and soft-tissue, and are consequently at increased risk for mesh exposure due to tissue atrophy [27]. The average age of our study cohort was $71.5 \pm 15.1$ years, which is in line with the hypothesis of why our patients encountered exposure and why titanium mesh may increase risk of complications. Additionally, any alloplastic foreign material implanted in the body is at subsequent risk of infection or subclinical infection, which may also increase complication risk. In large lesions where titanium mesh is necessary, the authors opt for free flap coverage, though the risk for complications remains high (Fig. 4). For these reasons, it may be advisable to investigate the success of scalp reconstruction with other underlying cranioplastic materials, including alloplastic materials, such as polyether ether ketone (PEEK) and porous polyethylene (MEDPOR), and autografts, such as bone, where appropriately indicated [30,31].

The authors recognize several limitations of this study. First, it is a retrospective study and may be prone to observer bias. Conclusions that we have drawn are not yet supported by prospective data. Methods of reconstruction may vary between individuals and practice, and we report only our institution's experience. The methods of reconstruction were impacted by the evolution of surgical techniques over the 16-year study period. One such example is the use of dermal matrices, which is a more recently developed technology, reflecting advances in materials and techniques of reconstructive surgery. As such, our current approach and guidelines provided also represent an evolution of approaches reflecting new technologies and evidence to practice. Furthermore, although we discuss the complications and analysis of each individual surgical method, the analysis of each method was underpowered for meaningful conclusions, and further studies examining each modality with larger patient samples will elucidate the specific indications, successes, and risk factors for each. Additionally, although it is possible that flap reconstruction may be advantageous when
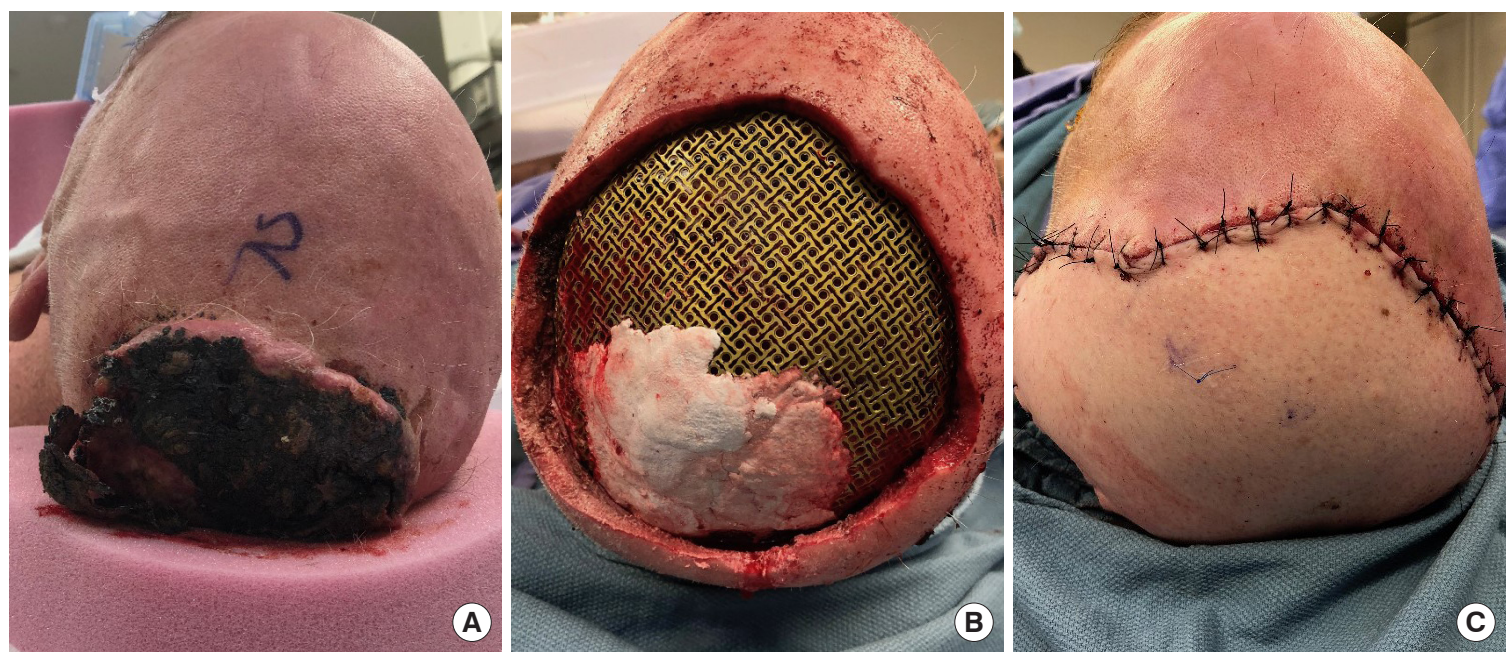

Fig. 4. An elderly male presents with a fungating lesion in the posterior scalp. (A) A fungating lesion preoperatively prior to wide resection. (B) Titanium mesh cranioplasty placed at the level of the calvarial defect following resection. (C) Complete postoperative free flap reconstruction overlying the titanium mesh. 
compared with other reconstructive methods in the setting of preoperative radiotherapy, larger numbers may be necessary to adequately study this hypothesis. Further, although radiation therapy did not offer a survival advantage in this patient population, its known ability to improve local control was not evaluated. The observation that preoperative radiation was predictive of mortality may also be related to underlying tumor stage and tumor grade. Despite these limitations, the presented findings support the hypothesis that preoperative radiation therapy for scalp lesions may be a major risk factor for complications. Further studies, including those of prospective design, are needed to more fully elucidate the factors that influence outcomes in the management of scalp malignancies and assess referral pathways to explore why patients with the same diagnosis may undergo different treatment strategies.

\section{NOTES}

\section{Conflict of interest}

No potential conflict of interest relevant to this article was reported.

\section{Ethical approval}

The study was approved by the Institutional Review Board of University of Pennsylvania (IRB No. 832418) and performed in accordance with the principles of the Declaration of Helsinki. Written informed consents were obtained.

\section{Patient consent}

The patients provided written informed consent for the publication and the use of their images.

\section{ORCID}

Michael G. Tecce https://orcid.org/0000-0002-7465-1354 Sammy Othman Jaclyn T. Mauch Shelby Nathan Estifanos Tilahun Robyn B. Broach Saïd C. Azoury Stephen J. Kovach https://orcid.org/0000-0002-8814-1048 https://orcid.org/0000-0002-1590-733X https://orcid.org/0000-0003-1545-8690 https://orcid.org/0000-0002-5571-7282 https://orcid.org/0000-0001-8507-3572 https://orcid.org/0000-0002-2263-4149 https://orcid.org/0000-0002-0308-5607

\section{REFERENCES}

1. Steiner D, Horch RE, Eyupoglu I, Buchfelder M, Arkudas A, Schmitz M, et al. Reconstruction of composite defects of the scalp and neurocranium: a treatment algorithm from local flaps to combined AV loop free flap reconstruction. World J
Surg Oncol 2018;16:217.

2. Ibrahim Z, Santiago GF, Huang J, Manson PN, Gordon CR. Algorithmic approach to overcome scalp deficiency in the setting of secondary cranial reconstruction. J Craniofac Surg 2016;27:229-33.

3. Desai SC, Sand JP, Sharon JD, Branham G, Nussenbaum B. Scalp reconstruction: an algorithmic approach and systematic review. JAMA Facial Plast Surg 2015;17:56-66.

4. Fischer JP, Sieber B, Nelson JA, Kovach SJ, Taylor JA, Serletti JM, et al. A 15-year experience of complex scalp reconstruction using free tissue transfer-analysis of risk factors for complications. J Reconstr Microsurg 2013;29:89-97.

5. Chao AH, Yu P, Skoracki RJ, Demonte F, Hanasono MM. Microsurgical reconstruction of composite scalp and calvarial defects in patients with cancer: a 10-year experience. Head Neck 2012;34:1759-64.

6. Swetter SM, Tsao H, Bichakjian CK, Curiel-Lewandrowski C, Elder DE, Gershenwald JE, et al. Guidelines of care for the management of primary cutaneous melanoma. J Am Acad Dermatol 2019;80:208-50.

7. Work Group; Invited Reviewers, Kim JYS, Kozlow JH, Mittal B, Moyer J, et al. Guidelines of care for the management of cutaneous squamous cell carcinoma. J Am Acad Dermatol 2018;78: 560-78.

8. Work Group; Invited Reviewers, Kim JYS, Kozlow JH, Mittal B, Moyer J, et al. Guidelines of care for the management of basal cell carcinoma. J Am Acad Dermatol 2018;78:540-59.

9. Newman MI, Hanasono MM, Disa JJ, Cordeiro PG, Mehrara BJ. Scalp reconstruction: a 15-year experience. Ann Plast Surg 2004;52:501-6.

10. Janus JR, Peck BW, Tombers NM, Price DL, Moore EJ. Complications after oncologic scalp reconstruction: a 139-patient series and treatment algorithm. Laryngoscope 2015;125:582-8.

11. Goessler UR, Bugert P, Kassner S, Stern-Straeter J, Bran G, Sadick $H$, et al. In vitro analysis of radiation-induced dermal wounds. Otolaryngol Head Neck Surg 2010;142:845-50.

12. Haubner F, Ohmann E, Pohl F, Strutz J, Gassner HG. Wound healing after radiation therapy: review of the literature. Radiat Oncol 2012;7:162.

13. Jasper KD, Holloway CL, DeVries KJ, Truong PT. Local relapse and survival outcomes in patients with scalp sarcoma: a retrospective study of 95 patients treated in a provincial cancer care institution over 25 years. Cureus 2019;11:e5236.

14. Ihara H, Kaji T, Katsui K, Miyake T, Waki T, Katayama N, et al. Single institutional experience of radiation therapy for angiosarcoma of the scalp without cervical lymph node metastases: Impact of concurrent chemoradiation with maintenance chemotherapy using taxanes on patient prognosis. Mol Clin Oncol 
2019;11:498-504.

15. Hata M, Wada H, Ogino I, Omura M, Koike I, Tayama Y, et al. Radiation therapy for angiosarcoma of the scalp: treatment outcomes of total scalp irradiation with X-rays and electrons. Strahlenther Onkol 2014;190:899-904.

16. Shin JY, Roh SG, Lee NH, Yang KM. Predisposing factors for poor prognosis of angiosarcoma of the scalp and face: systematic review and meta-analysis. Head Neck 2017;39:380-6.

17. Terakedis BE, Anker CJ, Leachman SA, Andtbacka RH, Bowen GM, Sause WT, et al. Patterns of failure and predictors of outcome in cutaneous malignant melanoma of the scalp. J Am Acad Dermatol 2014;70:435-42.

18. Xie C, Pan Y, McLean C, Mar V, Wolfe R, Kelly J. Impact of scalp location on survival in head and neck melanoma: a retrospective cohort study. J Am Acad Dermatol 2017;76:494-8.

19. Dundar Y, Cannon RB, Hunt JP, Monroe M, Suneja G, Hitchcock YJ. Radiotherapy regimens in patients with nonmelanoma head and neck skin cancers. Int J Dermatol 2018;57:441-8.

20. Harwood AR, Lawson VG. Radiation therapy for melanomas of the head and neck. Head Neck Surg 1982;4:468-74.

21. Wang HT, Erdmann D, Olbrich KC, Friedman AH, Levin LS, Zenn MR. Free flap reconstruction of the scalp and calvaria of major neurosurgical resections in cancer patients: lessons learned closing large, difficult wounds of the dura and skull. Plast Reconstr Surg 2007;119:865-72.

22. Stucker F, Ackermann D. Immunosuppressive drugs - how they work, their side effects and interactions. Ther Umsch 2011; 68:679-86.

23. Boots JM, Christiaans MH, van Hooff JP. Effect of immunosuppressive agents on long-term survival of renal transplant recipients: focus on the cardiovascular risk. Drugs 2004;64: 2047-73.

24. Eckardt A, Fokas K. Microsurgical reconstruction in the head and neck region: an 18-year experience with 500 consecutive cases. J Craniomaxillofac Surg 2003;31:197-201.

25. Cabraja M, Klein M, Lehmann TN. Long-term results following titanium cranioplasty of large skull defects. Neurosurg Focus 2009;26:E10.

26. Lee EI, Chao AH, Skoracki RJ, Yu P, DeMonte F, Hanasono MM. Outcomes of calvarial reconstruction in cancer patients. Plast Reconstr Surg 2014;133:675-82.

27. Maqbool T, Binhammer A, Binhammer P, Antonyshyn OM. Risk factors for titanium mesh implant exposure following cranioplasty. J Craniofac Surg 2018;29:1181-6.

28. Kwiecien GJ, Rueda S, Couto RA, Hashem A, Nagel S, Schwarz GS, et al. Long-term outcomes of cranioplasty: titanium mesh is not a long-term solution in high-risk patients. Ann Plast Surg 2018;81:416-22.

29. Othman S, Azoury SC, Tecce MG, Shakir S, Rios-Diaz AJ, Mauch JT, et al. Free flap reconstruction of complex oncologic scalp defects in the setting of mesh cranioplasty: risk factors and outcomes. J Craniofac Surg 2020;31:1107-10.

30. Oliver JD, Banuelos J, Abu-Ghname A, Vyas KS, Sharaf B. Alloplastic cranioplasty reconstruction: a systematic review comparing outcomes with titanium mesh, polymethyl methacrylate, polyether ether ketone, and Norian implants in 3591 adult patients. Ann Plast Surg 2019;82(5S Suppl 4):S289-4.

31. Goiato MC, Anchieta RB, Pita MS, dos Santos DM. Reconstruction of skull defects: currently available materials. J Craniofac Surg 2009;20:1512-8. 